\section{Estudo das variações dos níveis de retinol no colostro humano de parturientes a termo e pré-termo}

\section{Study of retinol level variations of human colostrum among parturient women with term and pre-term newborns}

Illana Louise Pereira de Melo 1 Karla Danielly da Silva Ribeiro 2 Roberto Dimenstein 3

1,2 Curso de Nutrição. Universidade Federal do Rio Grande do Norte, Natal, RN, Brasil.

3 Departamento de Bioquímica. Centro de Biociências. Universidade Federal do Rio Grande do Norte. Av. Senador Salgado Filho, 3000. Lagoa Nova. Natal, RN, Brasil. CEP: 59.072-970 E-mail: robertod@ufrnet.br

\begin{abstract}
Objectives: to analyze retinol levels variations in the colostrum of term and pre-term parturient women.

Methods: colostrum samples of 78 lactating women, half of which were mothers of premature newborns, were analyzed. The samples were manually extracted from one breast until $2.0 \mathrm{ml}$ were obtained. They were collected up to 72 hours after birth, during the morning hours and right before nursing. Retinol levels were determined by high-performance liquid chromatography and analyzed by the T-test for means comparison.

Results: the mean retinol values in the colostrums of women with term $(n=39)$ and pre-term newborns $(n=39)$ were 89,4 $\pm 46,1 \mu \mathrm{g} / \mathrm{dL}$ and 55,6 $\pm 27,7$ $\mu \mathrm{g} / \mathrm{dL}$, respectively. The difference of means was considered statistically significant at $(p<0,001)$. Human milk meets the need for Vitamin $A$ in the term newborn. However, for the premature newborn, it only supplied $66 \%$ of the needs.

Conclusions: results suggest that exclusive use of human milk does not meet the needs of pre-term newborn completely and that a Vitamin A supplement for the mother is necessary in order to reduce Vitamin A risk deficiency.
\end{abstract}

Key words Milk, human, Infant, premaure, Vitamin A

\section{Resumo}

Objetivos: analisar as variações dos níveis de retinol no colostro de parturientes a termo e pré-termo.

Métodos: foram analisadas amostras de leite de 78 lactantes, sendo metade delas mães de recémnascidos prematuros. As amostras de colostro foram obtidas por expressão manual de uma mama, até 72 horas após o parto, no turno vespertino e no início da mamada, até atingir um volume de 2,0 $\mathrm{ml}$. A determinação do retinol foi realizada por cromatografia líquida de alta eficiência e os resultados foram submetidos à análise estatística através do teste $t$ de Student.

Resultados: o valor médio do retinol no colostro de mães a termo $(n=39)$ e pré-termo $(n=39)$ foram, respectivamente, $89,4 \pm 46,1 \mu \mathrm{g} / \mathrm{dL}$ e 55,6 $\pm 27,7$ $\mu \mathrm{g} / \mathrm{dL}$. A diferença entre as médias foi estatisticamente significante $(p<0,001)$. O leite humano atende as necessidades de vitamina $A$ do recém-nascido a termo. Entretanto, o leite prematuro cobre apenas $66 \%$ das necessidades da criança prematura.

Conclusões: os resultados sugerem que as necessidades do recém-nascido pré-termo não podem ser completamente supridas por uso exclusivo de leite humano e que a suplementação materna de vitamina A pode reduzir os riscos de uma possível deficiência deste micronutriente.

Palavras-chave Leite humano, Prematuro, Vitamina $A$ 


\section{Introdução}

A vitamina A é um micronutriente fundamental para o crescimento, diferenciação e integridade do tecido epitelial, essencial nos períodos de gravidez e na primeira infância. ${ }^{1,2} \mathrm{~A}$ hipovitaminose $\mathrm{A}$ é um importante problema de saúde pública, sendo a principal causa de cegueira permanente entre crianças de países em desenvolvimento. ${ }^{2}$ Também contribui para o aumento significativo dos índices de morbi-mortalidade infantis associados a processos infecciosos. ${ }^{3}$ Muito do interesse na vitamina $\mathrm{A}$ em relação ao recém-nascido prematuro está no seu papel em ajudar a manter a integridade das membranas epiteliais, especialmente no pulmão e sua deficiência está associada com o aumento do risco de desenvolver a doença crônica pulmonar. 4,5

As necessidades nutricionais de vitamina A de uma criança prematura são maiores que em qualquer outra época da vida. Isto ocorre devido ao intenso catabolismo nas primeiras semanas após o nascimento, 6 junto ao baixo estoque de retinol no fígado ao nascer, as baixas concentrações de retinol plasmático e as baixas concentrações de proteínas carreadoras de retinol em relação aos recém-nascidos a termo. ${ }^{7}$ Além disso, o prematuro possui menor capacidade de digestão e absorção de lipídeos, por deficiência da lipase pancreática, o que resulta em diminuição da absorção de vitaminas lipossolúveis. ${ }^{8}$

Os benefícios do leite humano são bem conhecidos para crianças a termo, e dados sugerem que é especialmente útil para o prematuro. ${ }^{9}$ Entretanto, o leite prematuro apresenta algumas inadequações nutricionais do ponto de vista quantitativo, em face das necessidades fetais que deveriam ser supridas na vida intra-uterina através da transferência materno-fetal. 10

Segundo Mena e Milad, ${ }^{11}$ o leite humano não atende as necessidades de cálcio e fósforo para a mineralização óssea normal em bebês nascidos prematuramente e a diminuição do conteúdo de proteína ao longo da lactação pode necessitar de complementação para a perfeita alimentação do pré-termo. Quanto à vitamina $\mathrm{A}$, sua composição no leite de mães de lactentes prematuros e sua adequação como fonte desse nutriente para esse grupo ainda não foi totalmente elucidada e pouco se sabe sobre seus níveis no colostro humano prematuro. Dessa forma, o presente estudo tem o objetivo de analisar as variações dos níveis de retinol no colostro de parturientes a termo e pré-termo.

\section{Métodos}

\section{Amostra biológica}

Participaram do estudo 78 parturientes selecionadas por amostragem de conveniência e atendidas na Maternidade Escola Januário Cicco, em Natal, Rio Grande do Norte, Brasil, no período de novembro de 2003 a janeiro de 2004 Foram distribuídas, de acordo com a idade gestacional calculada através do método Capurro, em dois grupos: a termo $(n=39)$ e pré-termo $(n=39)$. Segundo Segre, 8 recém-nascido pré-termo é todo aquele que nasce antes de 37 semanas completas de idade gestacional. Mães que apresentavam gestações múltiplas, usuárias de suplementos com vitamina A, portadoras de intercorrências gestacionais ou de doenças crônicas foram excluídas do estudo. As amostras de colostro foram obtidas por expressão manual de uma mama, até 72 horas após o parto, no turno vespertino e no início da mamada, em um único momento até atingir um volume de 2,0 ml. O leite foi coletado em tubo de polipropileno envolto por papel alumínio, para impedir a degradação da vitamina A pela ação da luz, e conservada a $-20^{\circ} \mathrm{C}$ até o momento das análises. A amostra foi coletada uma única vez e padronizada para o início da mamada.

\section{Extração e determinação do teor de retinol}

O retinol no colostro foi extraído segundo o método de Giuliano et al.,12 e o extrato hexânico foi evaporado sob atmosfera de nitrogênio, em banho-maria a $37^{\circ} \mathrm{C}$. As amostras foram ressuspendidas em $1,0 \mathrm{ml}$ de metanol em grau de pureza para Cromatografia Líquida de Alta Eficiência (CLAE) e agitados por um minuto. O teor de retinol das amostras foi determinado por CLAE em Cromatógrafo LC-10 AD Shimadzu, acoplado a um Detector SPD-10 A Shimadzu UV-VIS e Integrador Chromatopac C-R6A Shimadzu com uma coluna LC Shim-pack CLC-ODS (M) 4,6 mm x $25 \mathrm{~cm}$. O cromatograma evoluiu nas seguintes condições: fase móvel metanol $100 \%$ e fluxo $1,0 \mathrm{ml} / \mathrm{min}$. A identificação e quantificação do retinol nas amostras foram estabelecidas por comparação com os tempos de retenção e as áreas dos respectivos padrões. As concentrações dos padrões foram confirmadas pelo coeficiente de extinção específico $(\varepsilon 1 \%, 1 \mathrm{~cm}=1780)$ em etanol absoluto $\mathrm{e}$ comprimento de onda de $325 \mathrm{~nm} .13$

O protocolo experimental do presente estudo foi submetido à análise do Comitê de Ética para Pesquisa em Humanos da Universidade Federal do Rio Grande do Norte obtendo parecer favorável (registro 102/03). 


\section{Análise estatística}

As diferenças estatísticas entre os grupos foram determinadas através do test $t$ Student, utilizando o programa Statistica 99 Edition (Copyright (C) 19841999 by StalSoft, Inc.) e foram consideradas significativas para $\mathrm{p}<0,05$.

\section{Resultados}

A determinação do valor médio de retinol das amostras de leite analisadas neste estudo demonstra uma diferença altamente significante na quantidade de retinol entre o leite colhido de mães a termo em relação àquele obtido de mães pré-termo. No primeiro, a média foi $89,4(\mathrm{DP}=46,1) \mu \mathrm{g} / \mathrm{dL}$ e naquele obtido de mães pré-termo este valor foi de $55,6(\mathrm{DP}=27,7) \mu \mathrm{g} / \mathrm{dL}, p=0,001$. (Figura 1)

\section{Figura 1}

Valores médios e desvio padrão de retinol no colostro de lactantes de parto a termo e pré-termo atendidas na Maternidade Escola Januário Cicco. Natal, Rio Grande do Norte, novembro de 2003 a janeiro de 2004.

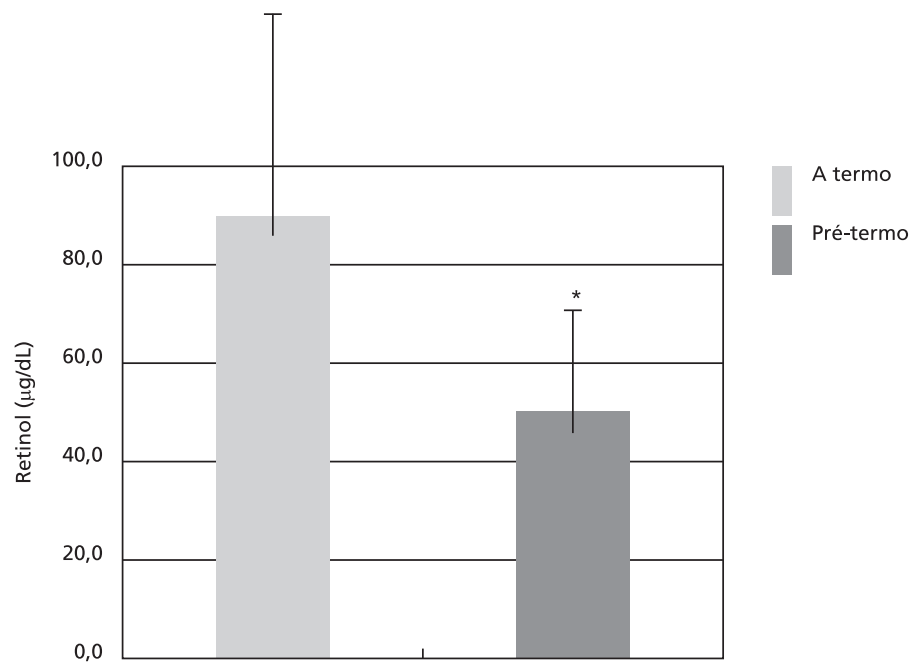

* $p<0,001$

\section{Discussão}

O valor médio de retinol no grupo a termo está de acordo com o encontrado por Dimenstein et al. ${ }^{14} \mathrm{e}$ Macias e Schuweigert 15 em mulheres brasileiras e cubanas, respectivamente.

Segundo Nascimento e Issler, 16 o conteúdo de vitamina A no leite humano é adequado para o recém-nascido. Entretanto, esse estudo mostra que o teor de vitamina A do leite prematuro não é apropriado para o recém-nascido visto que seus valores são menores quando comparados ao leite a termo. Como os recém-nascidos prematuros apresentam maiores necessidades desse micronutriente em relação ao recém-nascido a termo, é de fundamental importância um suporte adequado de vitamina A no leite materno. Isso reforça a idéia de que uma suplementação materna poderia reduzir os riscos de uma deficiência nutricional dessa vitamina.

De acordo com a Dietary Reference Intakes (DRIs) de 2001,17 a ingestão recomendada de vitamina A para crianças de zero a seis meses de idade é de $400 \mu \mathrm{g}$ por dia. Considerando um consumo médio diário de $500 \mathrm{~mL}$ de leite nas primeiras semanas de 
vida, como ressalta Ross e Philip, 18 os valores de retinol no leite a termo atende as necessidades da criança fornecendo $447 \mu \mathrm{g}$ por dia. Porém, em relação ao leite prematuro, os níveis de retinol no colostro não atendem as recomendações da DRI, pois só fornecem $278 \mu \mathrm{g}$ de retinol por dia. Essa situação se agrava quando são levadas em consideração as menores reservas na criança prematura e as maiores necessidades em relação ao recém-nascido a termo. De fato, segundo Segre, 8 a ingestão recomendada de vitamina A para o recém nascido prematuro é de $420 \mu \mathrm{g}$ por dia, e conforme verificado em nosso estudo, a ingestão foi de $278 \mu \mathrm{g} / \mathrm{dia}$. Assim o leite humano prematuro cobre apenas $66 \%$ das necessidades do recém-nascido.

Os resultados obtidos neste trabalho indicam que o retinol presente no leite prematuro tem uma concentração menor do que o leite a termo, sugerindo que as necessidades do recém-nascido pré-termo não podem ser completamente supridas por uso exclusivo de leite humano. Além disso, este estudo aponta informações importantes sobre o leite humano prematuro no sentido de direcionar pesquisas quanto à suplementação materna de vitamina $\mathrm{A}$, a fim de reduzir os riscos de uma possível deficiência nutricional dessa vitamina.

\section{Referências}

1. Azais-Braesco V, Pascal G. Vitamin A in pregnancy: requirements and safety limits. Am J Clin Nutr 2000; 71 [5 Suppl]: 1325-33.

2. Saunders C, Ramalho RA, Leal MC. Estado nutricional de vitamina A no grupo materno-infantil. Rev Bras Saúde Materno-Infantil 2001; 1: 21-9.

3. Mclaren DS, Frigg M. Manual de ver y vivir sobre los transtornos por deficiencia de vitamina A (VADD). Washington (DC): Organizacion Panamericana de la Salud, 1999.

4. Glasziou PP, Mackerras DEM. Vitaminas na gravidez e na primeira infância. Anais Nestlé 1996; 53: 37-47.

5. Darlow BA, Graham PJ. Vitamin A supplementation for preventing morbidity and mortality in very low birthweight infants. Cochrane Database Syst Rev 2002; 2: CD000501.

6. Ruffier CP, Ruffier JG, Pagani JR, Barreto APM, Braz MG. Neonatologia. In: Waitzberg DL. Nutrição oral, enteral e parenteral na prática clínica. São Paulo: Atheneu; 2000. p. 1051-86.

7. Vinagre RD, Diniz EMA. O leite humano e sua importância na nutrição do recém-nascido prematuro. São Paulo: Atheneu; 2002.

8. Segre CAM, Armellini PA, Marino WT. RN. São Paulo: Sarvier; 1995.

9. Lacerda EMA, Accioly E, Faria IG, Costa VM. Práticas em nutrição pediátrica. São Paulo: Atheneu; 2002.

10. Euclydes MP. Nutrição do lactente: base científica para uma alimentação adequada. Viçosa: Jard; 2000.

11. Mena PN, Milad MA. Variaciones en la composición nutricional de la leche materna. Algunos aspectos de importancia clínica. Rev Chil Pediatr 1998; 69: 116-21.
12. Giuliano AR, Neilson EM, Yap H, Baier M, Canfield LM. Quantitation of and inter/intra-individual variability in major carotenoids of mature human milk. J Nutr Biochem 1994; 5: 551-6.

13. Nierenberg DW, Nann SL. A method for determining concentrations of retinal, tocopherol, and five carotenoids in human plasma and tissue samples. Am J Clin Nutr 1992; 56: 417-26.

14. Dimenstein R, Simplício JL, Ribeiro KDS, Melo ILP. Retinol levels in human colostrum: influence of child, maternal and socioeconomic variables. J Pediatr 2003; 79: 513-8.

15. Macias C, Schuweigert FJ. Changes in the concentration of carotenoids, vitamin A, alpha-tocopherol and total lipids in human milk throughout early lactation. Ann Nutr Metab 2001; 45: 82-5

16. Nascimento MBR, Issler H. Breast feeding: making the difference in the development, health and nutrition of term and preterm newborns. Rev Hosp Clin Fac Med 2003; 58: $49-60$

17. Trumbo P, Schlicker S, Yates AA, Poos M. Dietary reference intakes for energy, carbohydrate, fiber, fatty acids cholesterol, protein and amino acids. J Am Diet Assoc 2002; 102: 1621-30.

18. Ross JS, Philip WJH. Contribution of breast feeding to vitamin A nutrition of infants: a simulation model. Bull World Health Organ 2003; 81: 80-6.

Recebido em 31 de março de 2004

Versão final apresentada em 26 de maio de 2004

Aprovado em 18 de julho de 2004 